\title{
Geometry effects at conductance quantization in quantum wires
}

\author{
G. Kiesslich, A. Wacker, and E. Schöll \\ Institut für Theoretische Physik, Technische Universität Berlin, Hardenbergstraße 36, D-10623 \\ Berlin, Germany
}

(physica status solidi, sceduled for Vol. 216/2, page R5 (1999))

(http://www.wiley-vch.de/berlin/journals/pss/rapid/contents/index.html\#99-061)

In Refs. $[1,2]$ the fabrication of quasi-onedimensional electronic systems (quantum wires) by cleaved edge overgrowth (CEO) in combination with a gate potential has been reported. Measured mean free paths [1] of about $10 \mu \mathrm{m}$ indicate that the electrons pass the wire ballistically. Therefore the Landauer formula suggests the linear-response conductance $G=$ $\frac{2 e^{2}}{h} M$, where $M$ is the number of transverse modes in the wire which can be reduced by increasing the gate potential $\left|V_{g}\right|$. In contrast, measurements [1,2] show plateaus below the theoretical value. Possible explanations may be based on either many particle interactions in the wire or geometry effects causing scattering at the ends of the wire. Our goal is to analyze the magnitude of those geometry effects neglecting interactions. Specifically, we investigate the influence of geometry and spatial potential landscape on the interference between wire states and contact states.

Our calculations were performed applying the method of equilibrium Green's functions following Ref. [3]. Within the discretization in tight binding approximation $(a=7.5 \mathrm{~nm})$ of the Hamiltonian and Dirichlet boundary conditions the Green's functions are finite matrices. The leads (source and drain) are treated in terms of self-energy. We use the effective mass $0.067 m_{e}$.

Fig. 1 shows the results of our calculations, where $V_{g}$ is applied over the whole range of the wire of length $L_{w}$ and width $b_{w}=53 \mathrm{~nm}$. In Fig. 1a (geometry as Fig. 1b) three plateaus appear because there are three transversal modes in the wire below the Fermi energy of 15 meV for $V_{g}=0$. Here, the width of the contacts, $b_{c}$, does not affect the transmission for $b_{c} \geq b_{w}$, thus we choose $b_{c}=b_{w}$. The solid line in Fig. 1a correspond to a rectangular potential step between contacts and wire. The oscillations in the conductance plateaus are well known as quantum mechanical transmission through a barrier and their frequency scales with the length of the wire. The dashed line in Fig. 1a follows from a calculation for a trapezoidal gate potential with a linear ramp over $30 \mathrm{~nm}$ at both ends of the wire. The oscillations almost disappear and almost complete quantization in every plateau is found. Thus we are still in the adiabatic regime [4] and no reflections at the boundary between contact and wire appear. However, in the solid curve the averaged plateau height of the lowest mode is clearly below the universal value in agreement with recent measurements in $\mathrm{V}$-groove wires [5]. If the potential changes abruptly in the transition between contacts and wire the mismatch between different states increases and there is a reduction in transmission for lower modes, which essentially affects the first jump at high $V_{g}$ while the subsequent jumps become closer to $2 e^{2} / h$.

Furthermore we considered an additional attracting potential $\phi$ (groove) close to the CEO interface (top boundary of Fig. 1d) which extends over the whole length $L_{w}+2 L_{c}$ and drops linearly along the $\mathrm{x}$ direction by $10 \mathrm{meV}$ on the scale of $53 \mathrm{~nm}$. The solid line in Fig. 1c refers to the U-shaped lead configuration (solid boxes for source and drain in Fig. 1d). In 
this case only two plateaus are observed: the lowest mode in the groove has an energy below the contact potential and is not accessible from the leads. If we change the configuration of leads (dashed boxes in Fig. 1d), three plateaus are observed again, see the dashed curve in Fig. 1c. Alternatively, one can obtain the same effect by placing some randomly distributed localized potentials in the groove regions, which act as scatterers. Thus, it is essential from which direction the electrons are injected into the device if this additional groove potential $\phi$ is present.

We have studied the geometry effects in cleaved edge overgrowth structures on the transmission through quantum wires. As long as the energy of the modes in the wire is above contact level, the junction between a wide contact and narrow wire has almost no influence on the transmission while a sharp potential step between contact and wire strongly affects the conduction. In contrast, modes with energies below the contact level may be difficult to access. Here the position of the external leads as well as the presence of scattering is of crucial importance. 


\section{REFERENCES}

[1] A. Yacoby, H.L. Stoermer, N.S. Wingreen, L.N. Pfeiffer, K. W. Baldwin, and K.W. West, Phys. Rev. Lett. 77, 4612 (1996).

[2] M. Rother, W. Wegscheider, M. Bichler, and G. Abstreiter, in Proceedings of the 24th Int. Conf. on the Physics of semiconductors, edited by D. Gershoni (World Scientific, Singapore, 1999).

[3] S. Datta, Electronic Transport in Mesoscopic Systems (Cambridge University Press, Cambridge, 1995); D. K. Ferry and S. M. Goodnick, Transport in Nanostructures (Cambridge University Press, Cambridge, 1997).

[4] L. Glazman, G. Lesovik, D. Khmel'nitskii, and R. Shekter, JETP Lett. 48, 238 (1988).

[5] D. Kaufman, Y. Berk, B. Dwir, A. Rudra, A. Palevski, and E. Kapon, Phys. Rev. B 59, 10433 (1999). 


\section{FIGURES}
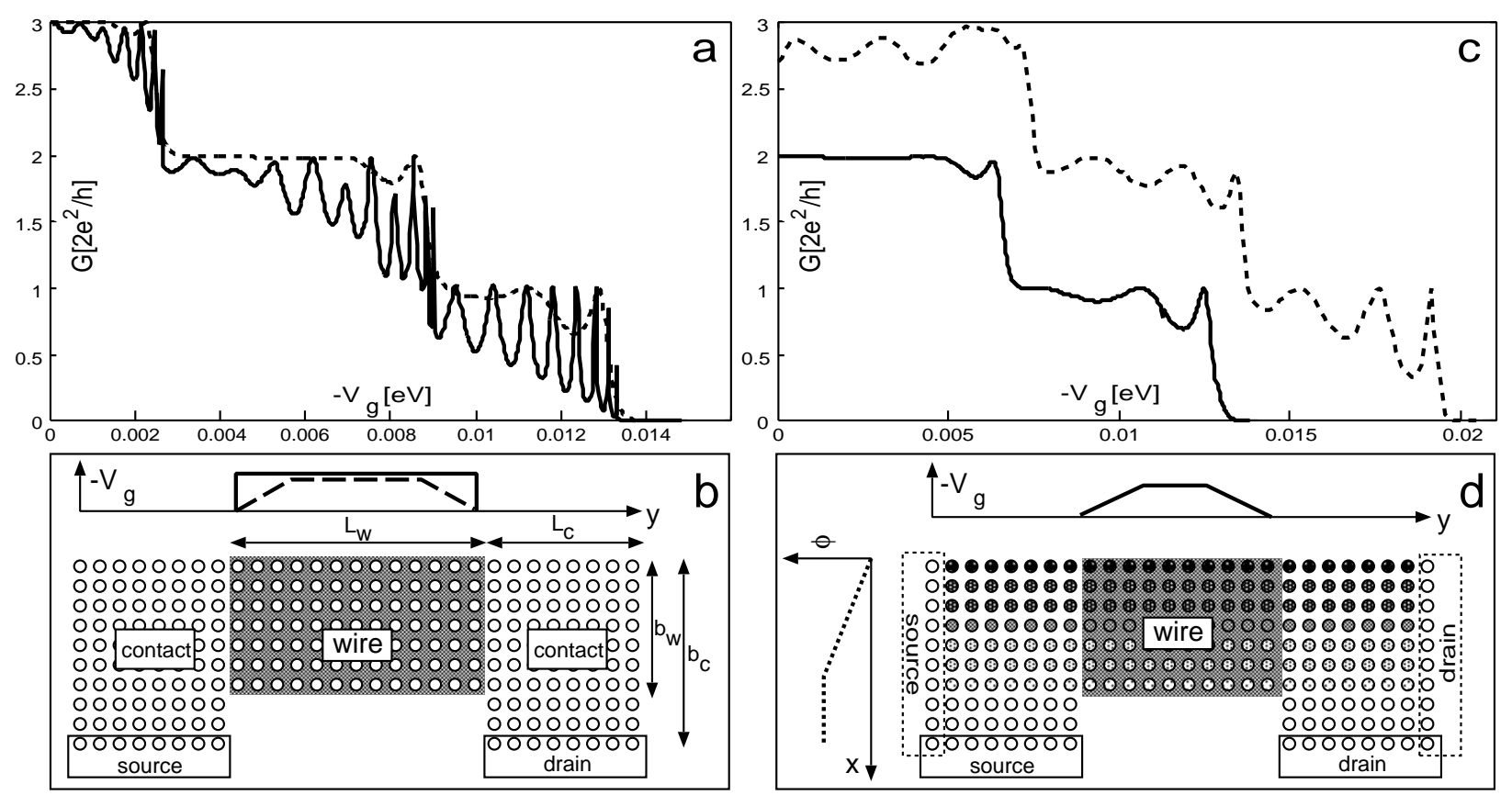

FIG. 1. Conductance $G$ versus gate voltage $V_{g}$ and scheme of CEO devices with different potential landscapes. (a,b) Rectangular (solid line) and trapezoidal (dashed line) gate potential in the wire (shaded region) for $L_{c}=300 \mathrm{~nm}, L_{w}=300 \mathrm{~nm}$. (c,d) Additional attracting potential $\phi$ close to the CEO interface for different lead configurations: U-shaped (solid source/drain box in (d) with $b_{c}=53 \mathrm{~nm}$, solid curve in (c)) and linear (dashed box in (d) with $b_{c}=150 \mathrm{~nm}$, dashed curve in (c)) for $L_{c}=300 \mathrm{~nm}, L_{w}=150 \mathrm{~nm}$. The lengths are not drawn to scale in (b,d). 